\title{
Urinary Incontinence Monitoring System Using Laser-induced Graphene Sensors
}

\author{
Anindya Nag, Subhas Mukhopadhyay \\ Faculty of Science and Engineering \\ Macquarie University \\ Sydney, Australia \\ Email: anindya1991@gmail.com
}

\author{
Jurgen Kosel \\ Computer Electrical and Mathematical Sciences and \\ Engineering Division \\ King Abdullah University of Science and Technology \\ Thuwal, Saudi Arabia
}

\begin{abstract}
This paper presents the design and development of a sensor patch to be used in a sensing system to deal with the urinary incontinence problem primarily faced by women and elderly people. The sensor patches were developed from laserinduced graphene from low-cost commercial polyimide (PI) polymers. The graphene was manually transferred to a commercial tape, which was used as sensor patch for experimentation. Salt solutions with different concentrations were tested to determine the most sensitive frequency region of the sensor. The results are encouraging to further develop this sensor in a platform for a fully functional urinary incontinence detection system
\end{abstract}

Keywords- Graphene, Polyimide, Laser-induced, Urinary incontinence, Salt concentration.

\section{INTRODUCTION}

Urinary incontinence is a condition where the loss of the capability to control the urinary bladder leads to accidental or involuntary loss of urine. This can cause severe inconvenience and embarrassment for affected people. The reasons for urinary incontinence can be high stress, damage caused in the vaginal region in women, effect on neurological conditions, kidney stones, removal of prostate gland in men, etc [1]. Pregnancy, menopause, and childbirth are some of the reasons, which causes urinary incontinence more among women than men [2]. Various approaches have been studied to monitor and treat incontinence. They include the placement of transobturator tapes in men [3] and women [4], using vaginal tapes for women [5, 6], and self-powered sensing systems [7]. The success of those methods is accompanied by certain disadvantages associated with them. The placement of the transobturator tapes involves surgery which needs specialized people and expensive equipments. The process of implanting vaginal tapes being implanted inside the urinary tract of a female is invasive, potentially painful and would cause disturbance to the patient. The self-powered system is somewhat ineffective, because the signal would only be transmitted over $500 \mathrm{~cm}$, which would not be enough for distant monitoring purposes. In addition, there are several risks associated with complications of the implanted device. The aim of this work is to design a low-cost system that can be operated by non-specialized person to detect urinary incontinence. The sensor should be incorporated with the underwear fabric making sure the patient is not conscious about the device and gets immediate attention when incontinence happens.

Sensors with flexible substrates [8-15] have been drawing attention recently, due to their advantages over the ones with rigid substrates in certain applications like higher mechanical flexibility, less weight, reduced cost or higher tolerance to strain. Flexible sensors are fabricated using different techniques and different raw materials are used to manufacture them. Polymers are attractive substrates to form flexible devices. Different ways to form flexible sensors [16, 17] have been studied for some time. In this paper, polyimide (PI) [18] was utilized to generate laser induced graphene, which is transferred to Kapton tape, forming electrodes. The high-speed operation, low-cost fabrication, and flexibility in terms of geometries are some of the advantages of using the laser cutting technique. Kapton has been used as substrates in many flexible devices $[19,20]$, due to its low cost, high flexibility and high temperature resistance.

Urine can be comprised of different constituents like urea, sodium chloride, potassium, creatinine and other compounds. Researchers have developed systems for salinity measurement based on the detection of sodium chloride [21, 22]. Thus, sodium chloride can be used as parameter to detect the presence of urine or sweat $[23,24]$. The average concentration of sodium values present in the urine of an adult is $460 \mathrm{ppm}$ [25]. Therefore, we conducted experiments with the laserinduced graphene sensors in solutions with different salt concentrations ranging from $300 \mathrm{ppm}$ to $600 \mathrm{ppm}$ to assess the possibility to use these sensor patches to develop an efficient system for the detection of urinary incontinence. The corrosion resistant nature of graphene [26] can be attributed as one of the major advantages of using graphene as electrodes for this application.

\section{FABRICATION OF GRAPHENE SENSORS}

${ }^{1}$ Low-cost polymer films (Zibo Zhongnan Plastics Co., Ltd.) were used as the raw material for this technique. The Polyimide (PI) films were attached to a glass substrate to keep it in place during laser ablation (Fig. 1(a)). A laser from Universal Laser Systems (Model: OLS 6.75 $\mathrm{CO}_{2}$ laser system,

\footnotetext{
${ }^{1}$ Research reported in this publication was supported by the King Abdullah University of Science and Technology (KAUST).
} 
laser spot diameter: 150 microns) was used to carry out the laser writing of the films. Corel Draw software was used for designing interdigitated electrodes with six pairs of fingers, each having a length and width of 500 microns and 100 microns, respectively. Graphene was obtained as the conductive material at the end of the process. During the laser writing, the $\mathrm{sp}^{3}$ hybridized carbon atoms of the polymer film were photo-thermally converted to $\mathrm{sp}^{2}$ hybridized atoms of graphene [18]. Three of the laser parameters namely power (W), speed $(\mathrm{m} / \mathrm{min})$ and $\mathrm{z}$-axis $(\mathrm{mm})$ was varied to obtain optimal results. The power defines the amount of power being exerted through the laser beam. The speed refers to the movement of the laser nozzle over the sample in $X-Y$ directions. The z-axis is used to control the focal point of the laser on the sample. The optimized values obtained for this process are $9 \mathrm{~W}, 70 \mathrm{~m} / \mathrm{min}$ and $1 \mathrm{~mm}$ for the power, speed and z-axis, respectively, which provided a good compromise between fabrication speed and pattern resolution. The laserinduced graphene was then transferred to Kapton tapes to use it as electrodes on the sensor patch (Fig. 1(b)). A manual pressure of around $150 \mathrm{kPa}$ was applied vertically onto the Kapton tapes which were placed on top of the induced graphene. The Kapton tape with the transferred conductive material was then pulled off carefully and used as the sensor patch. The conductivity of the induced graphene was around $11000 \mathrm{mS} / \mathrm{m}$. A difference of less than $20 \mathrm{mS} / \mathrm{m}$ was observed between before and after transferring the graphene.

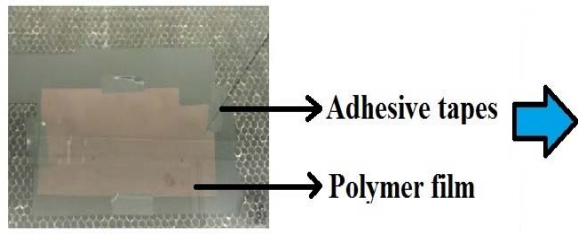

(a)

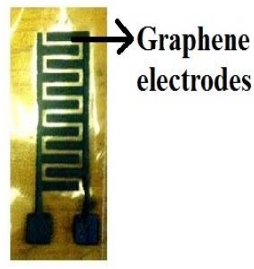

(b)
Fig. 1: Fabrication of the sensor. (a) A polymer film was attached to the glass substrate with adhesive tapes. (b) The developed graphene electrodes were transferred to a Kapton tape.

The electrodes of the developed sensor patches have an interdigital pattern with one sensing and one excitation electrode. The generated electric field fringes from one electrode to another due to the planar structure of the sensor. Thereby, the field penetrates through materials in close proximity, which are thus changing the distribution and magnitude of the field. The effects on the field can be studied to analyze the characteristics of the material under test. The penetration depth of the field can be varied by changing the spatial wavelength (distance between electrodes of the same polarity) of the electrodes. Non-destructive and in-situ measurements are some of the advantages of using these types of sensors.

\section{EXPERIMENTAL RESULTS}

Sodium Chloride (SA046- 500G) and de-ionized MilliQ ${ }^{\circledR}$ water (Resistance: $18.2 \mathrm{M} \Omega \mathrm{cm}$ and $\mathrm{pH}$ : 6.71) were used as the solute and solvent, respectively for the experiments with the sensor patches. Solutions of different concentrations were formed from $300 \mathrm{ppm}$ to $600 \mathrm{ppm}$. An initial stock solution of
$1000 \mathrm{ppm}$ was prepared by mixing $1 \mathrm{gm}$ of solute to $1000 \mathrm{ml}$ of solvent. The experimental solutions were then prepared from the stock by diluting it with de-ionized water to the desired concentrations. Electrochemical Impedance Spectroscopy was used to analyze the response of the sensor patches to the salt solutions. To this end, a HIOKI IM 3536 LCR high-precision tester was connected to the electrodes on the sensor patch using Kelvin probes. The sensor patch was attached to a wiring board with biocompatible tapes to keep it fixed inside of the solutions during experimentation.

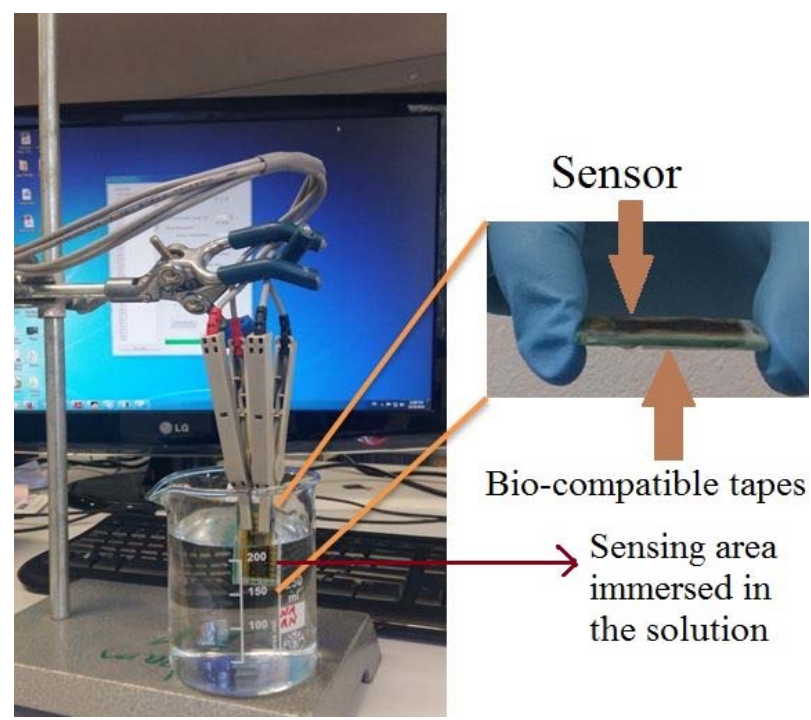

Fig. 2: Experimental setup showing the sensor patch immersed into the solution and the Kelvin probes connected to it for analyzing the response with an LCR meter.

Figure 2 shows the experimental setup. A USB cable was used to connect the LCR meter to the computer and collect the data via an automatic data acquisition algorithm. A sinusoidal voltage of $1 \mathrm{Vpp}$ amplitude and a frequency swept from $1 \mathrm{~Hz}$ to $10 \mathrm{kHz}$ was applied with the LCR meter. After the measurement with each sodium chloride concentration, the sensor was thoroughly washed with de-ionized water and dried for fifteen minutes in the oven (at $100^{\circ} \mathrm{C}$ ) before using it for the next experiment.

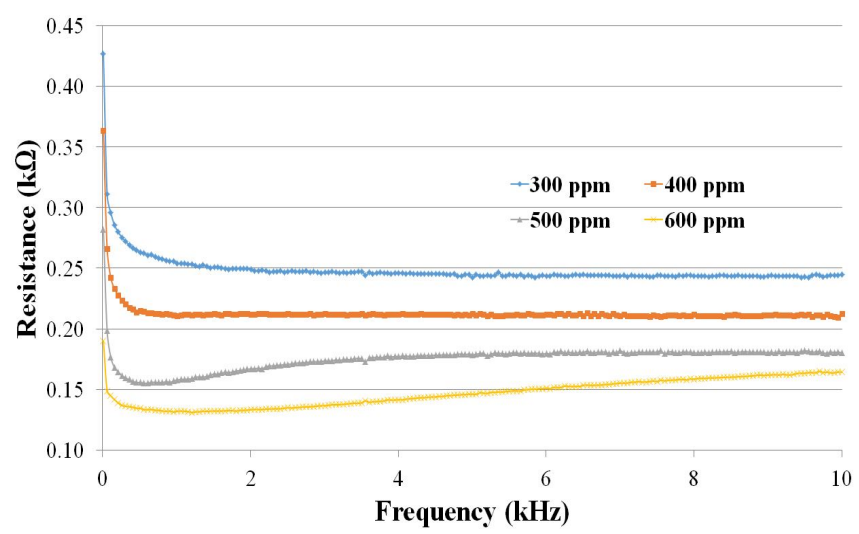

Fig. 3: Resistance response of the sensor patch for different concentrations of salt over frequency. 
Figure 3 depicts the response of the sensor patch to salt solutions of different concentrations in terms of resistance over the frequency. The double layer at the electrode causes a high resistance value at low frequencies, which is shorted at higher frequencies due to its capacitive nature, and only the constant resistance of the solution was measured. Further, as the salt concentration increases, the conductance of the solution increases; therefore, the measured resistance values decrease. Fig. 4 shows the resistance of the sensor as a function of the salt concentration at $4 \mathrm{kHz}$, where it is nearly frequency independent. It is seen that the response of the patch changes almost linearly with the salt concentration. The sensitivity value of these patches can be obtained from the slope of the curve and at these salt concentrations it is 0.3475 $\Omega / \mathrm{ppm}$.

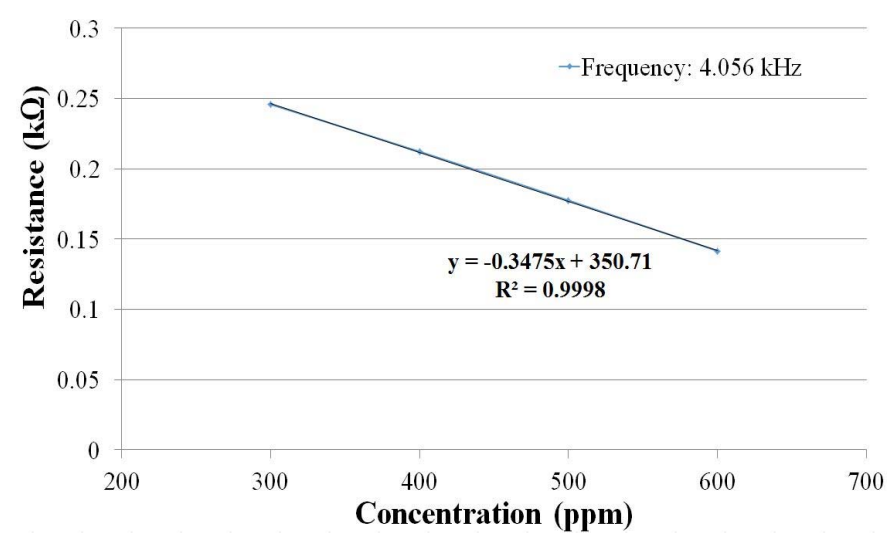

Fig. 4: Change in resistance with respect to different salt concentrations.

\section{CONCLUSION}

This paper shows the fabrication of a laser-induced graphene sensor for the detection of the salt concentration in water. The graphene was fabricated on PI films first and transferred to sticky tapes to act as electrodes. The simple fabrication process results in flexible sensor patches with interdigitated graphene electrodes on them. The advantages of these sensors include the high electrical conductivity of the electrodes $\left(\sim 10^{4} \mathrm{~S} / \mathrm{m}\right)$ and, the easy fabrication procedure. The sensor patch shows a linear response at concentrations ranging from $300 \mathrm{ppm}-600 \mathrm{ppm}$, with a sensitivity of $0.3475 \Omega / \mathrm{ppm}$, making this sensor suitable for monitoring urinary incontinence problems. The next step is to develop an embedded system, where data analysis will be applied in real-time. The sensor patch will be attached to the underwear of the patient to alert the monitoring unit. Once the sensor detects the presence of urine, the caretaker could be immediately informed to attend the patient.

\section{REFERENCES}

Causes of Urinary Incontinence. Available: http://www.nhs.uk/Conditions/Incontinenceurinary/Pages/Causes.aspx. Last accessed on 29.08.2017.

[2] L. N. Wood and J. T. Anger, "Urinary incontinence in women," Bmj, vol. 349, pp. 4531-4542, 2014.
P. Rehder and C. Gozzi, "Transobturator sling suspension for male urinary incontinence including post-radical prostatectomy," European urology, vol. 52, pp. 860-867, 2007.

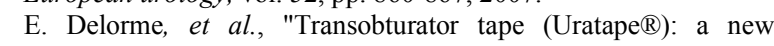
minimally-invasive procedure to treat female urinary incontinence," European urology, vol. 45, pp. 203-207, 2004.

C. Nilsson, et al., "Eleven years prospective follow-up of the tension-free vaginal tape procedure for treatment of stress urinary incontinence," International Urogynecology Journal, vol. 19, pp. 1043-1047, 2008.

J. de Leval, "Novel surgical technique for the treatment of female stress urinary incontinence: transobturator vaginal tape inside-out,"

European urology, vol. 44, pp. 724-730, 2003.
A. Tanaka, et al., "Self-powered wireless urinary incontinence sensor for disposable diapers," in Sensors, 2011 IEEE, 2011, pp. 1491-1494.

M. Y. Alnassar, et al., "Flexible Magnetoelectric Nanocomposites with Tunable Properties," Advanced electronic materials, vol. 2, 2016.

B. Li, et al., "Flexible magnetoimpedance sensor," Journal of Magnetism and Magnetic Materials, vol. 378, pp. 499-505, 2015.

A. Alfadhel and J. Kosel, "Magnetic nanocomposite cilia tactile sensor," Advanced Materials, vol. 27, pp. 7888-7892, 2015.

A. Nag, et al., "Wearable Flexible Sensors: A Review," IEEE Sensors Journal, 2017.

J.-Y. Baek, et al., "Flexible polymeric dry electrodes for the longterm monitoring of ECG," Sensors and Actuators A: Physical, vol. 143, pp. 423-429, 2008.

R. S. Dahiya and S. Gennaro, "Bendable ultra-thin chips on flexible foils," Sensors Journal, IEEE, vol. 13, pp. 4030-4037, 2013.

$\mathrm{S}$. Khan, et al., "Bendable piezoresistive sensors by screen printing MWCNT/PDMS composites on flexible substrates," in Microelectronics and Electronics (PRIME), 2014 10th Conference on Ph. D. Research in, 2014, pp. 1-4.

Y.-J. Yang, et al., "An integrated flexible temperature and tactile sensing array using PI-copper films," Sensors and Actuators A: Physical, vol. 143, pp. 143-153, 2008.

A. Arena, et al., "Flexible ethanol sensors on glossy paper substrates operating at room temperature," Sensors and Actuators B: Chemical, vol. 145, pp. 488-494, 2010.

C.-Y. Lee, et al., "Fabrication of micro sensors on a flexible substrate," Sensors and Actuators A: Physical, vol. 147, pp. 173176, 2008.

J. Lin, et al., "Laser-induced porous graphene films from commercial polymers," Nature communications, vol. 5, 2014.

$\mathrm{N}$. Lu, et al., "Metal films on polymer substrates stretched beyond

50\%," Applied Physics Letters, vol. 91, p. 221909, 2007.
A. Bedoya-Pinto, et al., "Flexible spintronic devices on Kapton," Applied Physics Letters, vol. 104, p. 062412, 2014.

J. Jonsson, et al., "Towards chip-based salinity measurements for small submersibles and biologgers," International Journal of Oceanography, vol. 2013, 2013.

H. C. Lim, et al., "Low Concentration Sodium Chloride Salinity Detection System," Sensors \& Transducers, vol. 141, p. 127, 2012. Sodium Chloride in Urine. Available: HTTP://WWW.WEBMD.COM/A-TO-Z-GUIDES/SODIUM-NAIN-URINE?PAGE=3. Last accessed on 29.08.2017.

Sodium Chloride concentration in Urine. Available: https://medlineplus.gov/ency/article/003601.htm Sodium Urine Test. Available: https://medlineplus.gov/ency/article/003599.htm Graphene paints a corrosion-free future. Available: http://www.manchester.ac.uk/discover/news/graphene-paints-acorrosion-free-future. Last accessed on 29.08.2017. 